\title{
Red in the face: Make the diagnosis
}

\author{
Mark A Miller MD
}

\begin{abstract}
A
43-year-old male presented to the emergency room with bilateral facial swelling and erythema. He had no past medical history; he was taking no regular medications and had no known allergies. He had been perfectly well until seven days earlier, when he noted the onset of fever and myalgias, with no other symptoms. He took occasional acetylsalicylic acid in an attempt to control the fever and aches, but his fever persisted and ranged from $37.2^{\circ} \mathrm{C}$ to $37.8^{\circ} \mathrm{C}$. He denied any chills, sweats, headache, tooth pain, chest pain, shortness of breath, urinary symptoms, diarrhea, joint pains or rash. He did feel tired, but continued to work. One day before his presentation, he noted the onset of diffuse redness over his entire nose, with progression to bilateral facial redness and swelling after $24 \mathrm{~h}$. There was no history of recent travel, insect bites or facial trauma.

On examination, the man appeared nontoxic and comfortable. Oral temperature was $38.8^{\circ} \mathrm{C}$, and other vital signs were normal. Impressive bilateral facial swelling and erythema were apparent (Figure 1), including bilateral periorbital involvement. The head and neck examination was otherwise normal, with no evidence of pharyngitis or sinus tenderness.
\end{abstract}

Departments of Microbiology, Sir Mortimer B Davis-Jewish General Hospital, and McGill University, Montreal, Quebec

Correspondence: Dr M Miller, Division of Infectious Diseases, 3755 chemin de la côte-Ste-Catherine, Montreal, Quebec H3T 1 E2. Telephone 514-340-8222, fax 514-340-7510, e-mailmmiller@lab.jgh.mcgill.ca

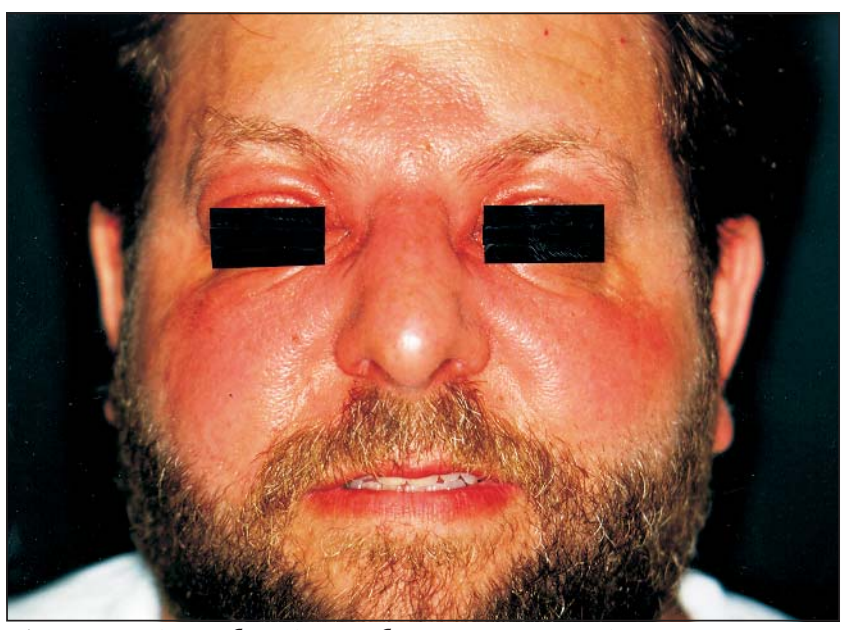

Figure 1)View of patient's face at presentation

Examination of the ears was normal. There was no adenopathy, and the rest of the examination was normal. Routine blood tests showed a normal hemoglobin of $145 \mathrm{~g} / \mathrm{L}$ and platelet count of $219 \times 10^{9} / \mathrm{L}$, with an elevated leukocyte count of $18.1 \times 10^{9} / \mathrm{L}$. Two sets of blood cultures were drawn.

What is the cause of this man's painless facial swelling, erythema and fever? What diagnostic test would you perform? 


\section{CLINICAL VIGNETTE}

Continued from page 383

\section{DIAGNOSIS}

Sinus X-rays were performed (single view in Figure 2), which showed complete opacification of both maxillary sinuses, air fluid levels in both frontal sinuses, opacification of the ethmoid sinuses and a questionable air fluid level in the sphenoid sinus. A computed tomography scan of the sinuses and orbits with infusion showed the soft tissue swelling of the face without extension into the orbits. The nasal cavity was completely occluded, with uptake of radiocontrast into the nasal, ethmoid and maxillary mucosa. The computed tomographic scan also showed complete occlusion of the maxillary sinuses by hypodense material, and partial occlusion of the sphenoid and frontal sinuses as well. Air fluid levels were again seen in the frontal sinuses. A diagnosis of facial erysipelas secondary to pansinusitis was made.

The patient was started on intravenous meropenem (Merrem, AstraZeneca, Mississauga, Ontario) with gradual improvement in the fever and facial swelling over the next four days. No sinus lavage was felt to be indicated, due to the adequate response to antibiotics alone. Blood cultures were negative, and he was changed to oral amoxicillin/clavulanic acid (Clavulin, SmithKline Beecham, Oakville, Ontario) and discharged home on day 5. A follow-up visit with the otolaryngologists was arranged.

\section{DISCUSSION}

Facial erysipelas used to be the most common form of erysipelas, now much more common in the lower extremity. It is almost always caused by Streptococcus pyogenes, but may be produced by beta-hemolytic streptococci of other types (ie, groups $\mathrm{C}$ or $\mathrm{G}$ ), as well as other bacteria in more rare circumstances. Preceding skin trauma or portals of entry are common, but facial erysipelas may also be caused by extension of orofacial foci such as odontogenic infections. Sinus infections as a source of facial erysipelas, without orbital involvement, are quite rare. The exact microbial etiology of facial erysipelas when caused by an underlying sinusitis is unknown, but may be polymicrobial in nature. Cultures of erysipelas are infrequently positive, even if performed by 'leading edge aspiration' of the rash. Bacteremia occurs less than $5 \%$ of the time. Therapy usually consists of antistreptococcal antibiotics (unless the condition is suspected to be caused by a polymicrobial

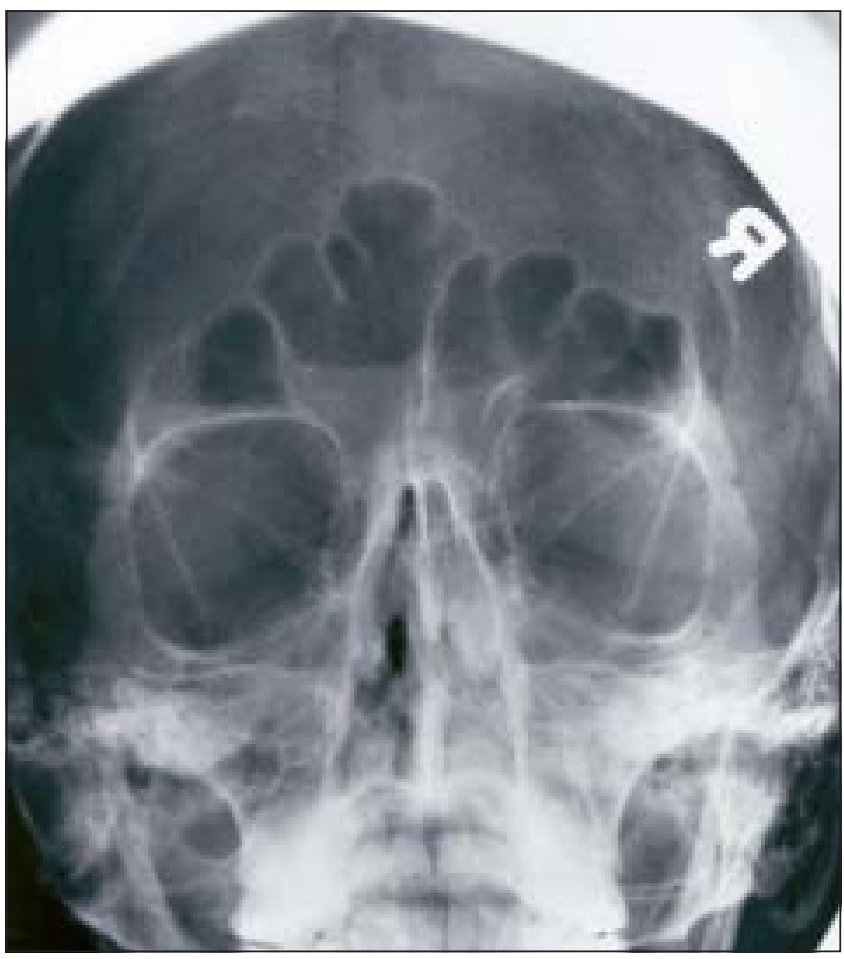

Figure 2) One view of the series of sinus radiographs taken at presentation

infection) and local wound care. Blistering and desquamation are common during the healing process.

The two unusual aspects of this patient's presentation are the sinus infection as the likely source of the erysipelas, and the lack of facial pain or pressure in the presence of such an extensive pansinusitis. When encountering a facial erysipelas without evidence of a portal of entry, a search for an underlying infectious source is mandatory. An examination of the teeth and sinuses are necessary. If no source is still evident, sinus X-rays should be completed in all such patients, regardless of symptoms.

\section{REFERENCES}

1. Jorup-Ronstrom C. Epidemiological, bacteriological and complicating features of erysipelas. Scan J Infect Dis 1986;18:519-24.

2. Evans FO, Sydnor JB, Moore WE, et al. Sinusitis of maxillary antrum. N Engl J Med 1975;293:735-9.

3. Ochs MW, Dolwick MF. Facial erysipelas: report of a case and review of the literature. J Oral Maxillofacial Surgery 1991;49:1116-20. 


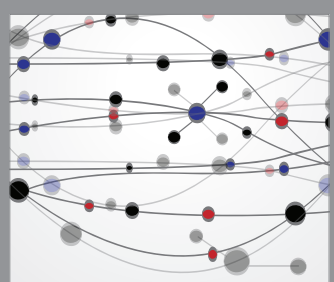

The Scientific World Journal
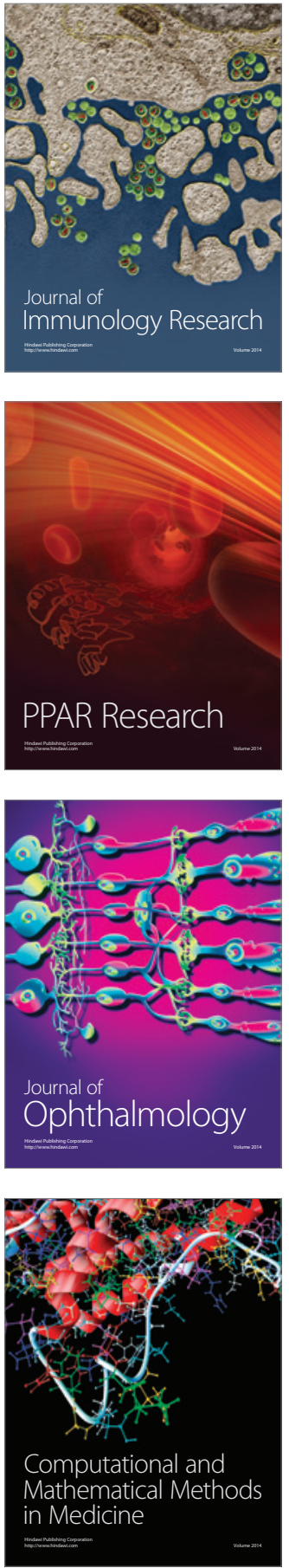

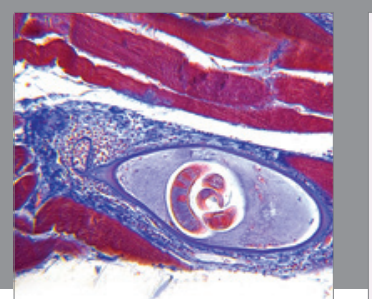

Gastroenterology Research and Practice

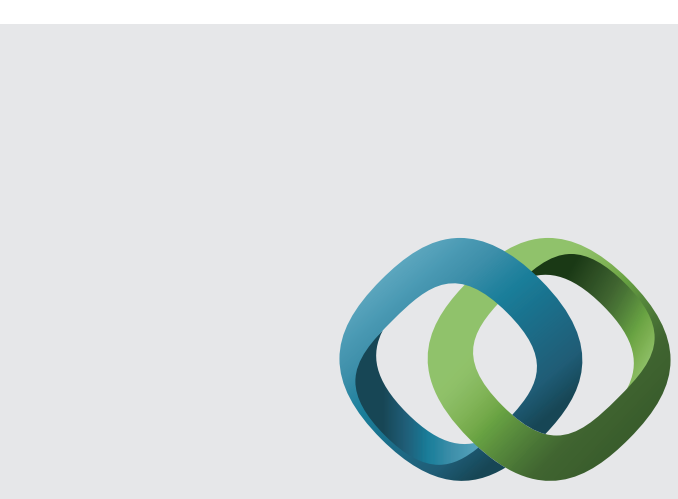

\section{Hindawi}

Submit your manuscripts at

http://www.hindawi.com
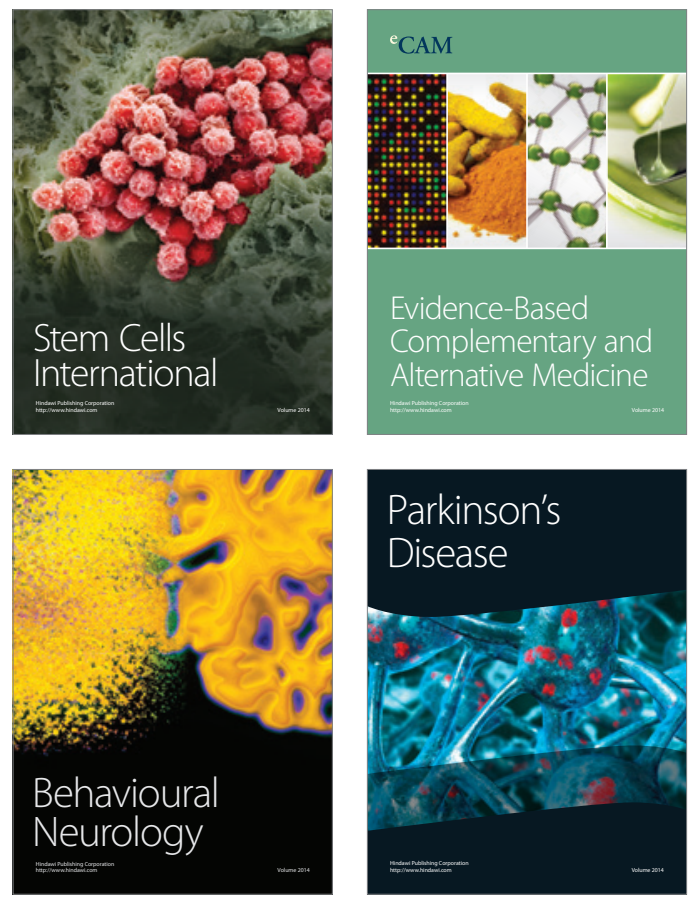
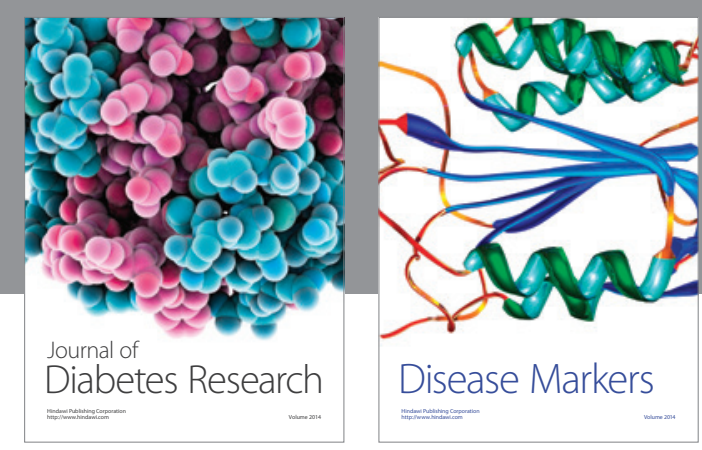

Disease Markers
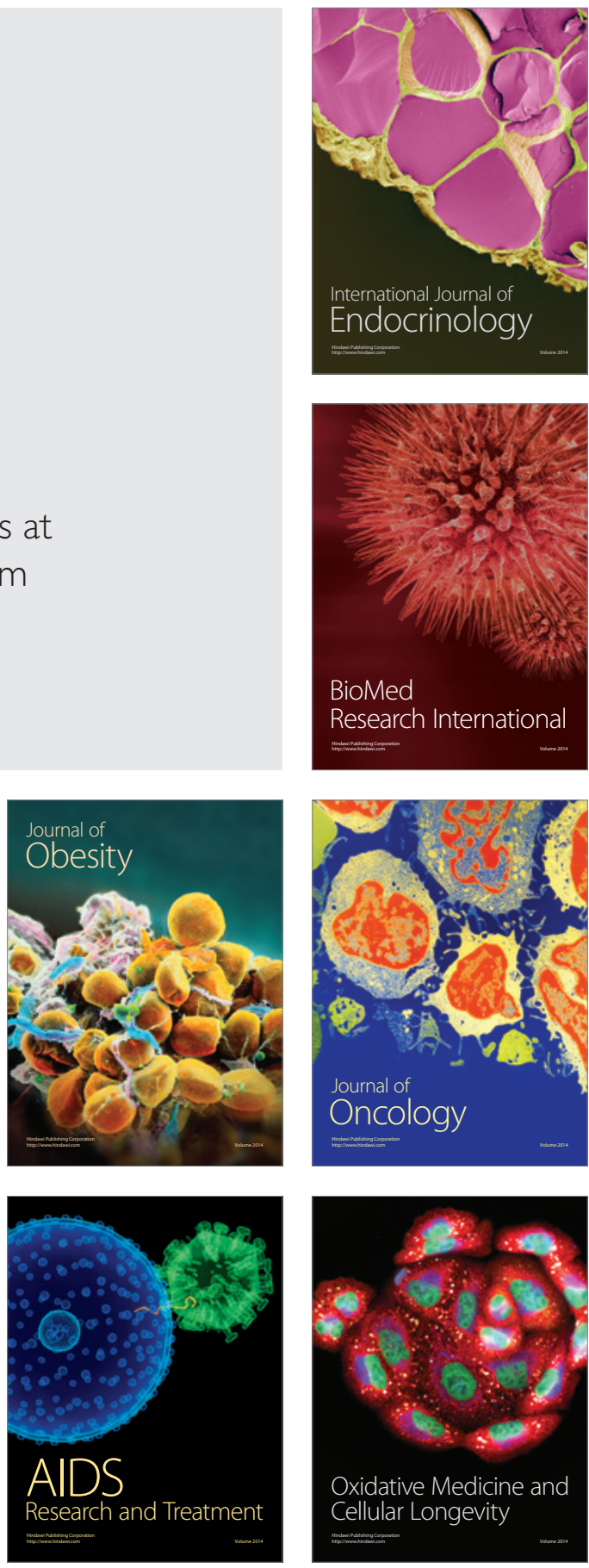